\title{
Novel Approach for Child and Adulthood Classification Based on Significant Prominent Binary Patterns of Local Maximum Edge (SPBPLME)
}

\author{
Rajendra Babu .Ch \\ SRK Institute of Technology, Vijayawada, 520008, India \\ Email: chikkalarajendra@gmail.com \\ Dr Sreenivasa Reddy. E, Dr Prabhakara Rao. B \\ ANU College of Engineering \& Technology, Guntur, 522510, India \\ JNTUK, Kakinada, 533003, India \\ Email: esreddy67@gmail.com,drbpr@rediffmail.com
}

\begin{abstract}
This paper derives a new procedure for age classification of facial image based on the local region of facial image. The local region of facial image is extracted from a Significant Binary Pattern of Local Maximum Edge (SBPLME). The SBPLME is generated by calculating the absolute value of local difference between the average of local $3 \times 3$ sub window pixel values and its neighbors instead of the center pixel value. In the case of Local Maximum Edge Binary Pattern (LMEBP) calculating the absolute value of local difference between the center pixel value of local $3 \times 3$ sub window and its neighbors. The proposed SBPLME can generate 512 (0 to 511) different patterns. The present paper utilized Prominent LBP (PLBP) on the proposed SBPLME. The PLBP contains the significant patterns of Uniform LBP (ULBP) and Non Uniform LBP (NULBP). Thus the derived Significant PLBP of Local Maximum Edge (SPBPLME) becomes an efficient image classification and analysis, which will have a significant role in many areas. The novelty of the proposed SPBPLME method is, it has shown excellent age classification results by reducing the overall dimension, thus reducing the overall complexity.
\end{abstract}

Index Terms-Age Classification, Prominent LBP, LMEBP, SBPLME, Maximum Edge.

\section{INTRODUCTION}

As humans, we are easily able to recognize a person's group i.e child and adult from an image of the person's face and are often able to be quite precise in this estimation. This ability has not been pursued in the computer vision community. In order to begin researching the issues involved in this process, this paper addresses the task of classification of facial image into either child or adult. The following section briefly explains about the existing approaches to analyze the facial image. The paper is organized into three four sections. The section 2 describes the existing methods. The proposed method is discussed in section 3 and results are discussed in sections 4 and conclusions are given in section 5.

\section{EXISTING METHIDS}

\section{A. Local binary patterns ( $L B P$ )}

Ojala et al. [1] was introduced the LBP operator for texture categorization and Analysis. The Local Binary Pattern (LBP) gives good results especially in texture analysis so that LBP mostly suitable in texture examination and its applications. The LBP operator has great tolerance against illumination changes and LBP calculation is also simple, due to this properties, it is well suitable for real-world applications like image texture analysis. The concept of LBP is also extended in applications such as face recognition and age classification [2, 3, 4].

To compute the LBP value at center pixel by subtracting its value from corresponding neighborhoods in the $3 \times 3$ sub window. The equation for calculating LBP values is shown in (1).

$$
\begin{aligned}
& \mathrm{LBP}=\sum_{\mathrm{i}=1}^{8} 2^{(\mathrm{k}-1)} \mathrm{f}(\operatorname{Img}(\mathrm{k})-\operatorname{Img}(\mathrm{l})) \\
& \mathrm{f}(\mathrm{z})= \begin{cases}0 & \mathrm{z}<0 \\
1 & \text { else }\end{cases}
\end{aligned}
$$

Where $\operatorname{Img}(l)$ denotes the center pixel gray value in $3 \times 3$ window, $\operatorname{Img}(k)$ is the neighboring pixels gray value. Figure 1 shows procedure for calculating LBP values from a given $3 \times 3$ sub image. The histograms of these patterns extract the existing edges in an image [5,6].

The eight bit LBP contains one of the two possible values, So that the combination of all the eight bits results in $2^{8}=256$ possible local binary patterns ranging from 0 to 255 . There is no distinctive way to construct the order of LBP on a $3 \times 3$ neighborhood. Figure 1 shows an example on how to generate the LBP value.

The Fig 1(a) represents the sample $3 \times 3$ sub window with grey values. The central pixel value is used as a threshold value in order to assign a binary value to its 
neighbors. Fig 1(b) shows the result of binary the $3 \times 3$ neighborhood based on threshold. The obtained binary values are multiplied by their corresponding weights. The weights matrix values are shown in as shown by Fig 1 (c). The resultant values matrix is given in Fig 1(d). The sum of the elements in resultant matrix i.e., Fig $1(\mathrm{~d})$ is treated as a LBP value. The LBP value in the below Figure 1 is 227. The central pixel in fig 1(a) 40 is replaced by the newly obtained new LBP value 227

\begin{tabular}{|l|l|l|}
\hline 63 & 28 & 45 \\
\hline 88 & $\mathbf{4 0}$ & 35 \\
\hline 67 & 40 & 21 \\
\hline \multicolumn{3}{|c|}{$(\mathrm{a})$} \\
\hline $2^{0}$ & $2^{1}$ & $2^{2}$ \\
\hline $2^{7}$ & & $2^{3}$ \\
\hline $2^{6}$ & $2^{5}$ & $2^{4}$ \\
\hline
\end{tabular}

(c)

\begin{tabular}{|c|c|c|}
\hline 1 & 0 & 1 \\
\hline 1 & & 0 \\
\hline 1 & 1 & 0 \\
\hline \multicolumn{3}{|c|}{ (b) } \\
\hline 1 & 0 & 4 \\
\hline 128 & & 0 \\
\hline 64 & 32 & 0 \\
\hline
\end{tabular}

(d)
Fig. 1. (a) Sample $3 \times 3$ sub window (b) Conversion values of Fig 1 (a) grey values into Binary sub Window (c) Representation of each neighborhood weight (d) The Values generated when Binary window in

Fig 1(b) is multiplied with Binary Weights shows in Fig 1(c).

\section{B. Local ternary patterns (LTP)}

The two valued LBP is extended to three valued code by Tan and Triggs [7] called local ternary patterns (LTP). The three values are generated by the following rules. If the gray value difference is zero then LTP value is 0 . If difference is less than zero then LTP value is -1 and otherwise +1 . i.e., the indicator $f(z)$ is replaced with three-valued function. Equation (3) and binary LBP code is replaced by a ternary LTP code as shown in Figure 2.

$$
f(z)= \begin{cases}-1 & x<0 \\ 0 & x=0 \\ +1 & x>0\end{cases}
$$

\begin{tabular}{|l|l|l|}
\hline 63 & 28 & 45 \\
\hline 88 & $\mathbf{4 0}$ & 35 \\
\hline 67 & 40 & 21 \\
\hline \multicolumn{3}{|c|}{ (a) } \\
\hline $2^{0}$ & $2^{1}$ & $2^{2}$ \\
\hline $2^{7}$ & & $2^{3}$ \\
\hline $2^{6}$ & $2^{5}$ & $2^{4}$ \\
\hline
\end{tabular}

(c)

\begin{tabular}{|c|c|c|}
\hline 1 & -1 & 1 \\
\hline 1 & & -1 \\
\hline 1 & 0 & -1 \\
\hline \multicolumn{3}{|c|}{ (b) } \\
\hline 1 & -2 & 4 \\
\hline 128 & & -8 \\
\hline 64 & 0 & -16 \\
\hline
\end{tabular}

Fig .2. (a) Sample $3 \times 3$ Neighborhood window with grey values (b) Ternary value $3 \times 3$ Neighborhood window of the fig 2 (a). (c) Binary weight matrix for a $3 \times 3$ window (d) Resultant matrix which is generated by multiplying fig 2 (b) with fig 2(c).

Figure 2 shows the scenario of calculating the LTP code on sample $3 \times 3$ window. The Fig 2 (a) represent the sample $3 \times 3$ neighborhood window with grey values which is same as consider in Fig 1(a). The central pixel value is considered as a threshold values in order to assign three values to its neighbors. Fig 2(b) shows the result of three valued $3 \times 3$ neighborhood window. The weighted matrix for calculating the LTP value is shown by Fig 2(c). The result matrix which is obtained by with elementary wise multiplying the matrix in Fig 2(b) with weighted matrix is represent in Fig 2(d). The sum of the elements in resultant matrix i.e., Fig 2(d) is treated as a LTP value. The LTP value generated in above Figure 2 is

171. The central pixel 40 in Fig 2(a) is replaced by the newly obtained LTP value i.e. 171.

\section{Local Maximum Edge Binary Pattern (LMEBP)}

The distribution of edges information in an image is extracted by the LMEBP [8]. In LMEBP, the edges are discriminated using directions (positive or negative direction). The directions are calculated between the center pixel values and its eight neighborhoods. The magnitude of the edges is not considered in LMEBP. Further, these edges are separate out into eight parts using the edge magnitudes. When compare with well-known LBP approach LMEBP captures more edge information.

\section{$D$. The draw backs of LBP, LTP and LMEBP}

After a careful and critical survey of the existing literature the present paper found that the LBP and LTP lose global spatial information completely. Moreover LBP and LTP does not provide better amount of distinguish information of the local structure and it has a various other disadvantages. The missing local information in the LBP, LTP is effectively represented and to overcome the edge sensitive problems of LMEBP, the present paper derived SBPLME.

The conventional LBP, LTP and LMEBP operator has some disadvantages and limitations:

- LBP is very perceptive to noise. A major difference between the central pixel and its environs is easily resulted by the slight fluctuations above or below the value of the central pixel with its neighbors.

- The LTP operator fails to detect large-scale facial image structures properly due to their small spatial support.

- The LBP utilized differences of adjacent pixels sign, by which it may lose significant local facial image information.

- The edges generated by LMEBP are sensitive and not efficient.

To address the above limitations and to preserve more edge information, the present paper derived SLEMBP. This SBPLME differs from the existing LMEBP and Local Binary Pattern (LBP). It extracts the most significant information based on division of edges in an image, generated from the difference between the average pixel values of a $3 \times 3$ neighborhood from each neighboring pixel. However in literature the LEMBP is proposed to captures edge information better than LBP and LTP. The proposed SLEMBP capture more edge information than LEMBP, which is evident from Figure 3 The Fig 3(a),Fig 3(c) and Fig 3(e) represents the edge images generated by LMEBP. The edge information of the proposed SBPLME captures more edge information than the existing LMEBP, which is evident from Fig 3(b), Fig 3(d) and Fig 3(f).

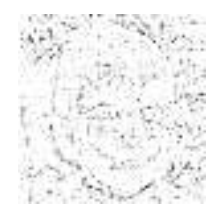

(a)

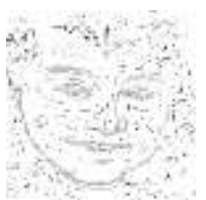

(b)

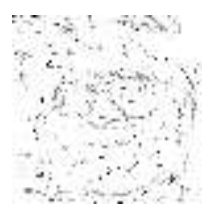

(c) 


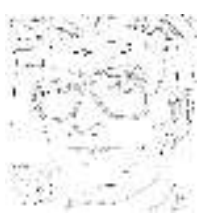

(d)

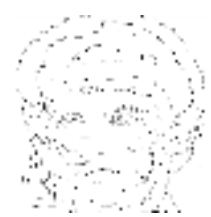

(e)

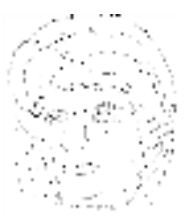

(f)
Fig. 3. Comparison of significant edge images between LMEBP and SBPLME. (a), (c) and (e) edge image generated by using LMEBP.(b) ,

(d) and (f) edge image generated by using the proposed SBPLME.

\section{PROPOSED METHOD}

\section{A. Age classification based on Significant Local} Maximum Edge using PBP (SPBPLME)

So many approaches are available for age group classification based on pattern approach [11-15] According to Ojala etal [1] LBP, which generates 256 patterns are grouped in to 59 uniform and 197 non uniform LBP patterns (NULBP). Many researchers have considered only uniform patterns (ULBP) for texture classifications due to their small numbers and claiming that most of the textures are dominated by only uniform
LBP's. But after a careful, critical and significant study on the existing literature, the present paper found that ULBP have some shortcomings: they discard some important texture information, suffer much from nonmonotonic illumination variation and do not describe the stochastic characteristics of texture efficiently and sensitive to noise. Further most of the extension works on LBP generates few or zero occurrences of zero transitions which are critical for ULBP. In such cases by considering ULBP generates a poor and insignificant classification. To overcome this, the present paper utilized Prominent LBP (PLBP) on the proposed SBPLME. The PLBP contains the significant patterns of ULBP and NULBP. Thus the derived Significant Local Maximum Edge PLBP (SPBPLME) becomes an efficient image classification and analysis.

The SPBPLME method consists of three stages. In the primary stage cropped facial image is obtained. The derivation of SBPLME is given in stage two. The evaluation of PBP on SBPLME is given in stage three. The block diagram of the SPBPLME method is shown in Fig 4.

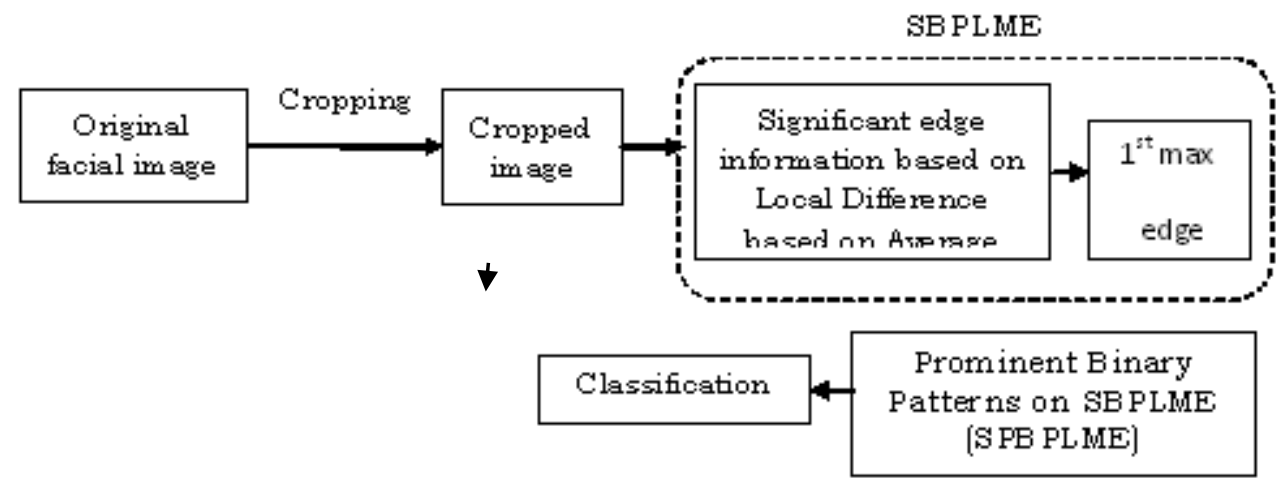

Fig. 4. Block diagram for child and adult classification using the proposed SPBPLME.

Stage One: The original facial image is cropped based on the location two eyes. Figure 5 shows an example of the original facial image and the cropped image.

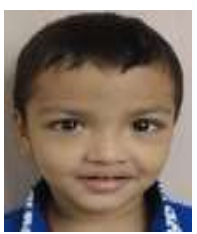

(a)

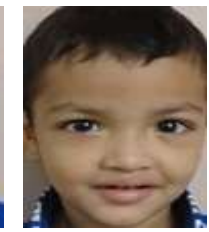

(b)
Fig .5. ( a) Existing Database facial image ,(b) cropped resultant image.

Stage Two: Derivation of SBPLME based on Local Difference using local average of $3 \times 3$ window pixels.

Significant Binary Patterns Local Maximum Edge(SBPLME) extracts the distribution of edges information in a facial image. The edges are distinguished using directions (positive or negative direction). The directions are calculated by finding the difference between the average value of the $3 \times 3$ window pixel values and its 8-neighbors as shown in Fig 6(a). The proposed SBPLME is different from LEMBP proposed by Subrahmanyam [8]. In LMEBP, the edges are discriminated using directions (positive or negative direction). The directions are calculated between the center pixel values and its eight neighborhoods. The magnitude of the edges is not considered in LMEBP. Further, these edges are separate out into eight parts using the edge magnitudes. When compare with well-known LBP approach LMEBP captures more edge information. The LMEBP is sensitive to noise and fails in capturing the significant edge information. The proposed SLEMBP overcomes this problem. The derivation of SBPLME is given below.

Step one: The Given facial Image (Img) is divided into $5 \times 5$ overlapped sub images.

Step two: For each facial sub-image of step one, calculate significant edges for each $3 \times 3$ overlapped window as represented in (4).

$$
\operatorname{Diff}(\mathrm{i})=(\operatorname{Img}(\mathrm{i})-\mathrm{AVG}) \mathrm{i}=1,2, \ldots, 8
$$

where AVG is the average value of the local $3 \times 3$ window pixels. 
Step Three: The local difference values of the 8neighboring pixel values are arranged in descending order (using absolute values) from first maximum edge value to eighth maxim edge value. If Diff(i) of the edge pixel $p_{i}$ is greater than zero, assign ' 1 ' to this corresponding neighboring pixel $\mathrm{p}_{\mathrm{i}}$ otherwise assign ' 0 ' as sown in (5).

$$
f(x)=\left\{\begin{array}{lr}
0 & x \geq 0 \\
1 & \text { otherwise }
\end{array}\right.
$$

By step two and three each $5 \times 5$ sub image window generates 9 overlapped $3 \times 3$ windows as shown in Fig 7 . And each $3 \times 3$ widow generates 8 bit binary values.

Step four: Place the binary sorted edge sequence of the each $3 \times 3$ window in a two dimensional edge array of 8 rows and 9 columns.

Step five: The first row of edge array is considered as the final edge sequence of SLEMBP because it contains the maximum edge of the $5 \times 5$ sub image. By this eight images are obtained on a given single input image. But the present paper considers first maximum edge values of SLEMBP and is used for finding prominent uniform patterns for child and adulthood classification, which are shown in Fig 3(b), Fig 3(d) and Fig 3(f).

The SBPLME computation for a $5 \times 5$ sub image is shown below. The Fig 6(a) contains a sample $5 \times 5$ sub image. The considered $3 \times 3$ window is also highlighted in the Fig 6(a). The average value obtained for the considered $3 \times 3$ window is 109 . Calculate the difference between Diif(i) value and its eight neighbors. The result is shown in Fig 6(b). The Fig 6(c) shows the binary sorted edge sequence of the $3 \times 3$ widow, arranged in a 2 $\mathrm{D}$ array. Based on the Diff(i) of the edge pixel $\mathrm{p}_{\mathrm{i}}$ is assigned a value ' 1 ' or ' 0 ' as represented in (6) also shown in Fig 6(c).

\begin{tabular}{|c|c|c|c|c|}
\hline 105 & 102 & 100 & 100 & 99 \\
\hline 101 & $\begin{array}{c}103 \\
(7)\end{array}$ & $\begin{array}{c}106 \\
(8)\end{array}$ & $\begin{array}{c}107 \\
(9)\end{array}$ & 106 \\
\hline 105 & $\begin{array}{c}110 \\
(6)\end{array}$ & $\begin{array}{c}113 \\
(1)\end{array}$ & $\begin{array}{c}111 \\
(2)\end{array}$ & 109 \\
\hline 107 & $\begin{array}{c}113 \\
(5)\end{array}$ & $\begin{array}{c}113 \\
(4)\end{array}$ & $\begin{array}{c}106 \\
(3)\end{array}$ & 104 \\
\hline 102 & 110 & 108 & 97 & 96 \\
\hline
\end{tabular}

Fig. 6 a). $5 \times 5$ window of the original image.

\begin{tabular}{|cc|cc|cc|}
\hline-6 & $\mathrm{a}$ & -3 & $\mathrm{~d}$ & -2 & $\mathrm{f}$ \\
\hline 1 & $\mathrm{~h}$ & \multicolumn{2}{|c|}{1} & 2 & $\mathrm{~g}$ \\
\hline 4 & $\mathrm{~b}$ & 4 & $\mathrm{c}$ & -3 & $\mathrm{e}$ \\
\hline
\end{tabular}

Fig.6. b). Difference values of $3 \times 3$ window.

\begin{tabular}{|c|c|c|c|c|c|c|c|}
\hline $\mathrm{a}$ & $\mathrm{b}$ & $\mathrm{c}$ & $\mathrm{d}$ & $\mathrm{e}$ & $\mathrm{f}$ & $\mathrm{g}$ & $\mathrm{h}$ \\
\hline-6 & 4 & 4 & -3 & -3 & -2 & 2 & 1 \\
\hline 0 & 1 & 1 & 0 & 0 & 0 & 1 & 1 \\
\hline
\end{tabular}

Fig.6. c). Sorted the difference values and assign 0 or 1 .

The working mechanism of SLEMBP for entire $5 \times 5$ window of Figure 6 is shown in Fig 7 and Fig 8. The nine windows of Fig 7 represent the Diff(i) i.e. the edge pixel value of the neighboring pixel. The Fig 6(b) represents the 2-D array arrangement of the maximum edge patterns arranged in sorted order from a to $h$ of the $3 \times 3$ window.

Stage Three: The calculation of the PBP on the proposed SBPLME (SPBPLME).

A LBP can generate 256 (0 to 255) different patterns. These patterns are grouped into 59 uniform and 197 non uniform LBP patterns. Originally patterns were detected by Ojala et al. [5, 6] in grey values. The fundamental properties of texture are these patterns. These patterns contain zero or two transitions (at most two one-to-zero or zero-to-one transitions). The patterns which contain zero or two transitions in the circular binary code defined in (6) are called "uniform" patterns. That is, the larger the uniformity value $U$ is, the more likely is that spatial transition occurs in the local pattern.

$$
\begin{gathered}
\mathrm{U}\left(\mathrm{LMEP}_{\mathrm{p}, \mathrm{r}}\right)=\quad\left|\mathrm{s}\left(\mathrm{q}_{\mathrm{p}-1}-\mathrm{q}_{\mathrm{c}}\right)-\mathrm{S}\left(\mathrm{q}_{\mathrm{o}}-\mathrm{q}_{\mathrm{c}}\right)\right| \\
+\left|\mathrm{S}\left(\mathrm{q}_{\mathrm{p}}-\mathrm{q}_{\mathrm{c}}\right)-\mathrm{s}\left(\mathrm{q}_{\mathrm{p}-1}-\mathrm{q}_{\mathrm{c}}\right)\right|
\end{gathered}
$$
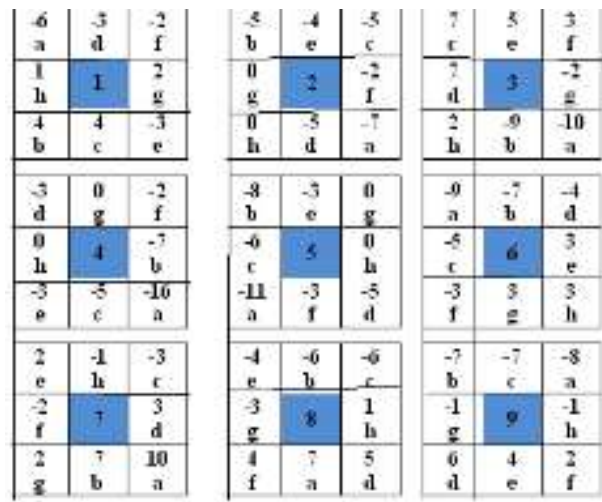

Fig . 7. Maximum Edge Pattern calculation of each center pixel in $3 \quad 3$ window

\begin{tabular}{c|cccccccccc} 
& 1 & 2 & 3 & 4 & 5 & 6 & 7 & 8 & 9 & value \\
\cline { 1 - 6 } $\mathrm{c}$ & 0 & 0 & 0 & 0 & 0 & 1 & 0 & 1 & 0 & 12 \\
$\mathrm{~b}$ & 0 & 0 & 0 & 0 & 0 & 0 & 1 & 0 & 0 & 4 \\
$\mathrm{c}$ & 1 & 0 & 1 & 0 & 0 & 0 & 0 & 0 & 0 & 64 \\
$\mathrm{e}$ & 1 & 0 & 1 & 0 & 0 & 0 & 1 & 1 & 1 & 71 \\
$\mathrm{f}$ & 0 & 0 & 1 & 0 & 0 & 1 & 1 & 0 & 1 & 77 \\
$\mathrm{~g}$ & 1 & 1 & 0 & 1 & 1 & 1 & 0 & 0 & 0 & 440 \\
$\mathrm{~h}$ & 1 & 1 & 1 & 1 & 1 & 1 & 0 & 1 & 0 & 506
\end{tabular}

Fig .8. Binary values of 9 centers $1^{\text {st }}$ maximum edge to $8^{\text {th }}$ maximum edge

Many researchers have considered only ULBP patterns for texture classifications due to their small numbers and claiming that most of the textures are dominated by only uniform LBP's. But after a careful, critical and significant study on the existing literature, the present study found that ULBP have some shortcomings: they discard some important texture information by not considering any one of the 197 non uniform patterns, suffer much from non- 
monotonic illumination variation and do not describe the stochastic characteristics of texture efficiently and sensitive to noise. To overcome this, the present paper utilized 'Prominent LBP' (PLBP) The PLBP contains some of the non uniform patterns and some of the uniform patterns that derive significant information.

The 9-bit SBPLME generates a total of 512 patterns ranging from 0 to 511 . Thus considering such a huge size of patterns for classification purpose is a very complex task and tedious process.

For example, patterns 0 (bitwise 000000000) and 511 (bitwise 111111111) have a $U$ value of 0 while patterns 1 , 2, 4, 8, 16, 32, 64 and 128, 256 (bitwise 000000001, 000000010 , etc.) have a $U$ value of 2 as there are exactly two $0 / 1$ or $1 / 0$ transitions in the bitwise representation. A UBP contains only this pattern that consists of 0 or two transitions. This represents a total number $\mathrm{P} \times(\mathrm{P}-1)+3$ distinct uniform patterns on $\mathrm{p}=9$ bits (i.e., 75 of $3 \times 3$ neighborhood) of the $2 \mathrm{P}$ un-rotated patterns that can occur in a sub image of $5 \times 5$ of SBPLME. This results a total number $437(512-75=437)$ non uniform patterns in a sub image of $5 \times 5$ of SBPLME. Non uniform patterns fall in to a large category of patterns.

The present study found that most of the extension works on LBP have very few or negligible occurrences of zero transitions and a good number occurrences of four transitions. Based on this prominent uniform (PLBP) are defined in the literature by. V.Vijaya kumar et al. PLBP are those patterns of LBP with exactly two or four bitwise transitions ( i.e $U=2$ 4). (two or four one-to-zero, zeroto-one transitions) That is the prominent LBP considers sub set of non-uniform and uniform patterns. The present method utilized the concept of PLBP on SBPLME. Thus SPBPLME contains a total of 97 patterns in a sub image of $5 \times 5$ of SBPLME. To have a exact and correct child and adult categorization, the present method considered sum of the rate of recurrence of SPBPLME on the facial image.

\section{RESULTS AND DISCISSIONS}

The proposed scheme established a database from two sources. The first source from FG-Net aging base which consists of 1002 human face images various ages from zero age to 90 years. The second source 600 face images downloaded from Google Images. The total images in the database are 1602 sample facial images. Some of the images in FG-NET database are shown in Fig 9. In the current proposed method it is assumed that age is from 0 to 16 years are treated as child and age is above 16 years treated as adult. In the proposed method the sample images are grouped into either child or adult based on frequency occurrences of SPBPLME.

The sum of rate of recurrence of SPBPLME patterns on child and adult facial images are list out in Table 1 and 2 respectively. The Table 1 and Table 2 also shows sum of rate of recurrence of zero transitions (which are part of ULBP) and ULBP on SBPLME. The table clearly indicates and establishes the fact that zero transitions need not necessary to count because they contain negligible or zero occurrences. The classification graph of child and adult categorization based on the proposed method is shown in Fig 10. Based on the frequency of occurrence of PBP on the proposed SLEMBP an algorithm is derived to efficiently classify the adulthood, which is shown below.

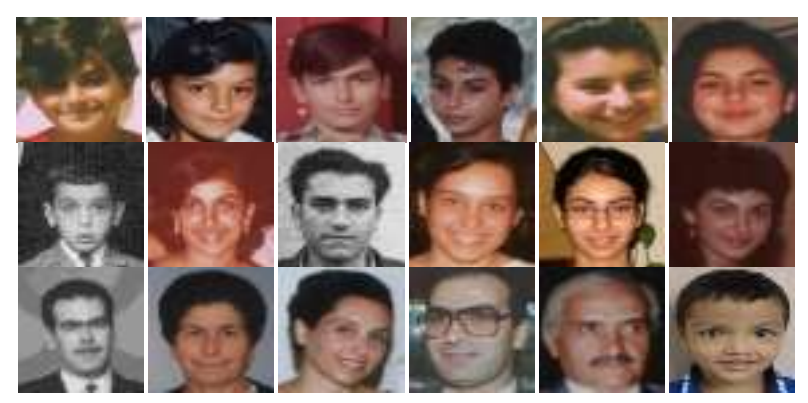

Fig. 9. Sample images in FGNet Aging data base

Table 1. Sum of frequency of occurrence of various patterns on SBPLME on child facial images

\begin{tabular}{|c|c|c|c|c|}
\hline SNO & $\begin{array}{l}\text { Image } \\
\text { name }\end{array}$ & $\begin{array}{l}\text { Frequency } \\
\text { occurrences } \\
\text { of ZERO } \\
\text { transitions on } \\
\text { SBPLME }\end{array}$ & $\begin{array}{c}\text { Frequency } \\
\text { occurrences } \\
\text { of UBP on } \\
\text { SBPLME }\end{array}$ & $\begin{array}{c}\text { Frequency } \\
\text { occurrences } \\
\text { of PBP on } \\
\text { SBPLME }\end{array}$ \\
\hline 1 & 011A07 & 0 & 1828 & 6531 \\
\hline 2 & 011A05 & 0 & 1922 & 9742 \\
\hline 3 & 010A10 & 0 & 2245 & 8893 \\
\hline 4 & 010A09 & 0 & 2221 & 8695 \\
\hline 5 & $001 \mathrm{~A} 16$ & 0 & 1628 & 9737 \\
\hline 6 & 010A07b & 0 & 1784 & 9944 \\
\hline 7 & 002A16 & 0 & 1942 & 9808 \\
\hline 8 & 001A14 & 0 & 2183 & 9947 \\
\hline 9 & 009A16a & 0 & 2700 & 9312 \\
\hline 10 & 009A14 & 0 & 2672 & 9898 \\
\hline 11 & 009A13 & 0 & 2579 & 9211 \\
\hline 12 & 009A11 & 0 & 2896 & 9656 \\
\hline 13 & 008A16 & 0 & 2911 & 9099 \\
\hline 14 & $008 \mathrm{~A} 13$ & 0 & 2825 & 9742 \\
\hline 15 & 008A12 & 0 & 2494 & 9238 \\
\hline 16 & 010A06 & 0 & 2721 & 9721 \\
\hline 17 & 010A05 & 0 & 2614 & 8714 \\
\hline 18 & 010A04 & 0 & 2665 & 8331 \\
\hline 19 & 010A01 & 0 & 2384 & 8224 \\
\hline 20 & 009A09 & 0 & 1793 & 6641 \\
\hline 21 & 009A05 & 0 & 2759 & 9854 \\
\hline 22 & 008A08 & 0 & 2998 & 9996 \\
\hline 23 & 008A12 & 0 & 4056 & 9551 \\
\hline 24 & 008A13 & 0 & 2340 & 8071 \\
\hline 25 & 008A16 & 0 & 2670 & 9890 \\
\hline
\end{tabular}

Algorithm 1: Age group categorization based on rate of recurrence of SPBPLME

if $($ FREQUENCY $($ SPBPLME $)<10000)$

display ("facial image is CHILD")

else

display ( "facial image is ADULT")

end 
Table 2. Sum of frequency of occurrence of various patterns on SBPLME on adult facial images

\begin{tabular}{|c|c|c|c|c|}
\hline $\mathrm{SNO}$ & $\begin{array}{l}\text { Image } \\
\text { name }\end{array}$ & $\begin{array}{l}\text { Frequency } \\
\text { occurrences } \\
\text { of ZERO } \\
\text { transitions } \\
\text { on SBPLME }\end{array}$ & $\begin{array}{l}\text { Frequency } \\
\text { occurrences of } \\
\text { UBP on } \\
\text { SBPLME }\end{array}$ & $\begin{array}{l}\text { Frequency } \\
\text { Occurrences } \\
\text { of PBP on } \\
\text { SBPLME }\end{array}$ \\
\hline 1 & 004A21 & 0 & 3259 & 10186 \\
\hline 2 & 002A29 & 0 & 4356 & 10328 \\
\hline 3 & 002A26 & 0 & 2935 & 10980 \\
\hline 4 & $002 \mathrm{~A} 23$ & 0 & 1870 & 11383 \\
\hline 5 & $002 \mathrm{~A} 23$ & 0 & 1685 & 12735 \\
\hline 6 & 001A29 & 0 & 3465 & 13943 \\
\hline 7 & 001A33 & 0 & 3387 & 14048 \\
\hline 8 & 001A40 & 0 & 2864 & 12465 \\
\hline 9 & 001A43a & 0 & 2778 & 13031 \\
\hline 10 & $001 \mathrm{~A} 43 \mathrm{~b}$ & 0 & 2168 & 14886 \\
\hline 11 & 002A20 & 0 & 3255 & 13297 \\
\hline 12 & $002 \mathrm{~A} 26$ & 0 & 2401 & 14672 \\
\hline 13 & 002A29 & 0 & 2240 & 13678 \\
\hline 14 & 002A36 & 0 & 2827 & 13198 \\
\hline 15 & 003A49 & 0 & 2544 & 16230 \\
\hline 16 & 003A51 & 0 & 3066 & 12708 \\
\hline 17 & 003A58 & 0 & 2979 & 13265 \\
\hline 18 & 003A60 & 0 & 2874 & 12497 \\
\hline 19 & 003A61 & 0 & 2878 & 13373 \\
\hline 20 & 004A19 & 0 & 2821 & 13649 \\
\hline 21 & 004A26 & 0 & 3536 & 14177 \\
\hline 22 & 004A40 & 0 & 3520 & 15067 \\
\hline 23 & 004A48 & 0 & 3261 & 13747 \\
\hline 24 & 004A51 & 0 & 3421 & 15247 \\
\hline 25 & 004A53 & 0 & 3749 & 16962 \\
\hline
\end{tabular}

From the Table 1 and Table 2 it is clearly apparent that it is not possible to segregate the human's age as adult and childhood accurately based on UBP and zero patterns on proposed SBPLME. From this it is clearly evident that UBP fails in classification where as PBP results a high classification rate. If prominent binary patterns count is less than 10000 then the facial image is treated as child and the facial image is treated as adult. The classification percentage of two datasets is shown in Table 3.

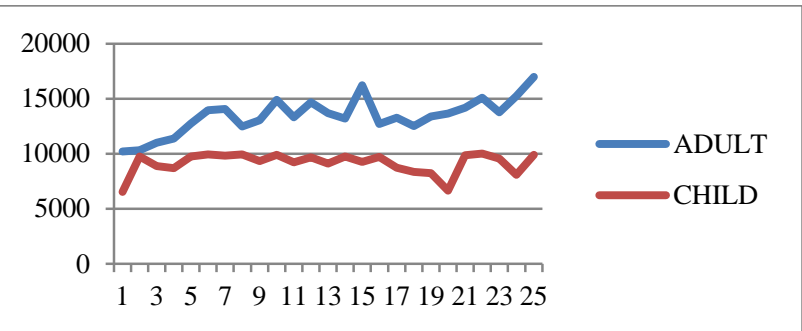

Fig.10. Classification graph of child and adult classification based on the proposed SPBPLME.

Table 3. Classification results of the proposed SPBPLME method in two datasets

\begin{tabular}{|c|c|c|}
\hline Image Dataset & FG-NET database & Google database \\
\hline Child & $97.4 \%$ & $95.8 \%$ \\
\hline Adult & $96.4 \%$ & $94.3 \%$ \\
\hline
\end{tabular}

\section{A. Comparison of the proposed method with Existing Methods}

The efficiency of the proposed method is compared with an existing "Morphological Primitive Patterns with Grain Components on LDP approach" method [9] and "geometric properties" approach [10] methods. This paper evaluated mean classification rates for zero transitions of uniform patterns, uniform binary patterns and prominent binary patterns on SBPLME. The percentage of classification rates are listed in Table 4 and categorization graph is shown in Fig 11. The percentage of categorization rates of the proposed method and other existing methods $[10,9]$ are listed in Table 5. The Table 5 clearly indicates that the proposed method yields better categorization rate when compared with the existing methods. Fig 12 shows the comparison chart of the proposed method with the other existing methods of Table 5.

Table 4. $\%$ mean categorization rates for zero, uniform and semi uniform patterns on SBPLME

\begin{tabular}{|c|c|c|c|}
\hline $\begin{array}{c}\text { Image } \\
\text { Dataset }\end{array}$ & $\begin{array}{c}\text { ZERO transitions } \\
\text { on SBPLME }\end{array}$ & $\begin{array}{c}\text { UBP on } \\
\text { SBPLME }\end{array}$ & $\begin{array}{c}\text { PBP on } \\
\text { SBPLME }\end{array}$ \\
\hline Child & $0 \%$ & $87.14 \%$ & $96.83 \%$ \\
\hline Adult & $0 \%$ & $65.17 \%$ & $95.42 \%$ \\
\hline
\end{tabular}

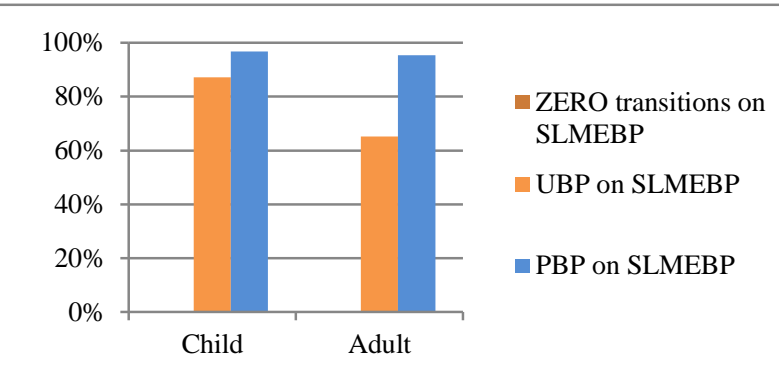

Fig. 11. Comparison graph of proposed method on zero transitions, UBP and PBP

Table 5. \% mean classification rates for proposed SPBPLME method and other existing methods.

\begin{tabular}{|c|c|c|c|}
\hline $\begin{array}{c}\text { Image } \\
\text { Dataset }\end{array}$ & $\begin{array}{c}\text { "Morphological } \\
\text { Primitive Patterns } \\
\text { with Grain } \\
\text { Components on } \\
\text { LDP" approach }\end{array}$ & $\begin{array}{c}\text { "Geometric } \\
\text { properties } \\
\text { Approach" }\end{array}$ & $\begin{array}{c}\text { Proposed } \\
\text { Method } \\
\text { SPBPLME }\end{array}$ \\
\hline Child & $92.17 \%$ & $91.04 \%$ & $96.83 \%$ \\
\hline Adult & $93.37 \%$ & $92.71 \%$ & $95.42 \%$ \\
\hline
\end{tabular}

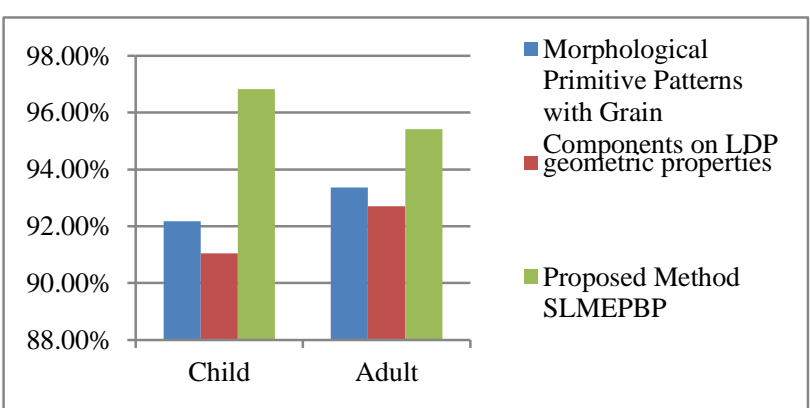

Fig .12. Comparison graph of proposed method with other existing methods 


\section{CONCLUSIONS}

The paper developed a new trend for the child and adult classification using prominent patterns on SBPLME of human face. The existing LMEBP is sensitive to noise and suffers in establishing the prominent and significant edges. The proposed SBPLME overcomes this, by establishing significant edges. The proposed PLBP on the proposed SBPLME overcomes the overall complexity of classification algorithm. The PBP on the proposed SBPLME shows the supremacy and efficiency over ULBP on SBPLME. The present method is tested on both FG-NET aging database and Google Images. The performance of the present scheme is more for the FGNET aging database than Google Images.

\section{ACKNOWLEDGMENT}

The authors would like to express their gratitude to Sri B.S.Appa Rao, Chairman of SRK Foundation, B.S.Krishna Secretary and Correspondent of SRK Foundation, Dr P.Venkata Narasaiah, Director of SRK Institution of Technology and Dr D.Haritha Department Head for providing necessary infrastructure and facilities for doing research work.

\section{REFERENCES}

[1] T Ojala, M PietikaÈinen, and D Harwood published a paper entitled "A Comparative Study of Texture Measures with Classification Based on Feature Distributions" in the Journal of Pattern Recognition, Year: 1996 volume: no : 29 Pages 51 to 59.

[2] M Chandra Mohan, V Vijaya Kumar and B Sujatha published a paper entitled "Classification of child and adult based on geometric features of face using linear wavelets," in the Journal of Signal and Image Processing, volume no: 1 and issue no:3, yeAr : 2010 Pages:211 to 220.

[3] " Texton Based Shape Features on Local Binary Pattern for Age Classification", Chandra Sekhar Reddy et al.; ,IJIGSP 2012, 7, 54-60

[4] "Novel method of adult age classification using linear wavelet transforms", Chandra Mohan, VijayaKumar V., Venkata Krishna V., IJCSNS, pp:1-8, 2010.

[5] "Texture Classification by Multi-Predicate Local Binary Pattern Operators", Maeenpaeae T., PietikaÈinen M., and Ojala T., Proc. $15^{\text {th }}$ Int'l Conf. Pattern Recognition, vol.3, pp.951-954, 2000.

[6] "Robust texture classification by subsets of local binary patterns", MaeenpaeaeT., Ojala T., PietikaÈinen M., and Soriano M., Proc. 15th Int'l Conf. Pattern Recognition, vol.3, pp. 947-950, 2000.

[7] "Enhanced local texture feature sets for face recognition under difficult lighting conditions" by Tan X, Triggs B, IEEE Transactions on Image Processing 19 (6) (2010) $1635-1650$

[8] "Local maximum edge binary patterns: A new descriptor for image retrieval and object tracking" by Subrahmanyam M, Maheshwari R P, Balasubramanian R , International Journal of Signal Processing 92 (2012) pages: 1467-1479

[9] "Morphological Primitive Patterns with Grain Components on LDP for Child and Adult Age Classification" by Sujatha B, Vijaya Kumar Vijaya Kumar, Rama Bai M,
International Journal of Computer Applications (0975 8887) Volume 21- No.3, May 2011

[10] "Classification of child and adult based on geometric features of face using linear wavelets", Chandra Mohan M., Vijaya Kumar V., Sujatha B., IJSIP, vol.1, Iss.3, pp:211$220,2010$.

[11] Jangala. Sasi Kiran, V. Vijaya Kumar and B. Eswara Reddy. "Age classifications based on second order image compressed and fuzzy reduced grey level (SICFRG) model," International Journal on Computer Science and Engineering (IJCSE), Vol. 5 No. 06, pp.481 -492, Jun 2013.

[12] Sasi Kiran, Dr. V. Vijaya Kumar and V V Harichandana, "An Effective Age Classification Using Topological Features Based on Compressed and Reduced Grey Level Model of the Facial Skin," I.J. Image, Graphics and Signal Processing, Vol. 6, No. 1, pp. 9-17, November 2013.

[13] Gorti Satyanarayana Murty, J Sasi Kiran and Dr. V. Vijaya Kumar, " Facial Expression Recognition based on Features Derived from the Distinct LBP and GLCM," International Journ al of Image, Graphics and Signal Processing(IJIGSP), MECS Publisher, Hong Kong, Vol. 6, No. 2, pp. 68 - 77, January 2014.

[14] Gorti Satyanarayana Murty, Dr. V. Vijaya Kumar and A. Obulesu, "Age classification based on simple LBP transitions," International Journal on Computer Science and Engineering (IJCSE), Vol. 5 No. 10, pp. 885 - 893, October 2013

[15] P S S V ravi Kumar, P Kiran Kumar Reddy, and V Vijaya Kumar, "Age Classification Based On Features Extracted From Third Order Neighborhood Local Binary Pattern", ictact journal on image and video processing, november 2014, volume: 05 , issue: 02

Authors' Profiles

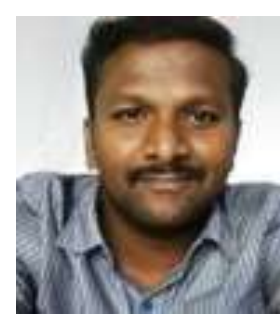

Rajendra Babu Ch:Received the B.Tech degree in Computer Science \& Engineering from Jawaharlal Nehru Technological University, Hyderabad, India in 2005, M.Tech. degree in Computer Science and Engineering from Acharya Nagarjuna University, India in 2008, and registered for Ph.D. in Computer Science and Engineering at Jawaharlal Nehru Technological University under the guidance of Prof. E. Srinivasa Reddy and Prof. B.Prabhakara Rao. His research interests include Image Processing.

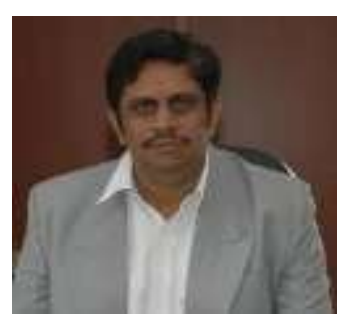

Sreenivas Reddy E: Received the B.Tech degree in Electronics \& Communication Engineering from Nagarjuna University, India in 1988, M.S. degree from Birla Institute of Technology and Science, India in 1997, M.Tech degree in Computer Science from Visveswaraiah Technological University, India in 2000 and Ph.D in computer science from Acharya Nagarjuna University, India in 2008. He is the senior member of IEEE and presented 11 papers in international conferences and 6 journal papers. Presently working as principal in ANU college of Engineering. His research interest includes image processing, biometrics and pattern recognition. 


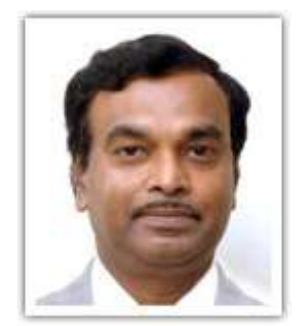

Prabhakara Rao B: Has more than 28 years of experience in teaching and 20 years of $\mathrm{R} \& \mathrm{D}$. $\mathrm{He}$ is an expert in Signal Processing \& Communications .He produced 5 PhD's and guiding $25 \mathrm{PhD}$ scholars. He held Head of the Department, in JNTU College of Engineering. Presently working as Rector in JNTU Kakinada. He published more than 85 technical papers in national and International journals and conferences.

How to cite this paper: Rajendra Babu .Ch, Sreenivasa Reddy. E, Prabhakara Rao. B,"Novel Approach for Child and Adulthood Classification Based on Significant Prominent Binary Patterns of Local Maximum Edge (SPBPLME)", International Journal of Information Technology and Computer Science(IJITCS), vol.7, no.6, pp.30-37, 2015. DOI: 10.5815/ijitcs.2015.06.04 